\title{
SURFACES IN $G(1,5)$ WITH ONE APPARENT DOUBLE POINT
}

\author{
JOSÉ CARLOS SIERRA*
}

\begin{abstract}
In this work we characterize the smooth surfaces that can be embedded in the Grassmannian of lines $G(1,5)$ with one apparent double point, i.e., such that the general linear projection to $G(1,3)$ produces just one double point. The result is that such a surface must be either a rational scroll of degree 4 or 5 or a Del Pezzo surface of degree 6 or 7 .
\end{abstract}

\section{Introduction}

It is a well known result that every $r$-dimensional smooth subvariety $X \subset \mathrm{P}^{N}$ can be isomorphically projected to $\mathrm{P}^{2 r+1}$ from a general $(N-2 r-2)$-linear subspace. The further projection to $\mathrm{P}^{2 r}$ from a general point produces a finite number $\delta(X)$ of double points.

In the case of smooth surfaces in $\mathrm{P}^{5}$, Severi's theorem states that the only one that can be isomorphically projected to $\mathrm{P}^{4}$ (i.e., with $\delta(X)=0$ ) is the Veronese surface (see [9]). In the same paper, Severi claims that the smooth surfaces with one apparent double point (i.e., with $\delta(X)=1$ ) are the rational normal scrolls of degree 4 and the Del Pezzo surface of degree 5. In the proof he assumes that surfaces with one apparent double point cannot have a large family of trisecant lines. This fact was first remarked by Ciliberto and Sernesi, and the proof was recently obtained by F. Russo in [8], completing the theorem. A related problem, studied also by Severi in [9], is to find the smooth surfaces in $\mathrm{P}^{4}$ without apparent triple points, i.e., the union of its trisecant lines does not fill $\mathrm{P}^{4}$. The result was stated by A. Aure in [4], showing that the quintic elliptic scroll is the only smooth surface in $\mathrm{P}^{4}$ without apparent triple points that do not lie on a quadric hypersurface.

The aim of this work is to prove a similar result for smooth surfaces in Grassmannians of lines, in the spirit of [3], [1], [10] or [2]. To be precise, we study the restriction to a surface of the linear projection $G(1,5) \rightarrow G(1,3)$

\footnotetext{
* The author was supported by a Pre-Doc Fellowship of MEC. Partial support came from the Research Project BFM2000-0621 from the Spanish Ministry of Science and Technology, as well as from the OMATS program.

Received March 9, 2004.
} 
induced, in the obvious way, by a linear projection $\mathrm{P}^{5} \rightarrow-\rightarrow \mathrm{P}^{3}$. It is known that the only one that can be isomorphically projected is the Veronese surface, i.e., $\mathrm{P}^{2}$ embedded by the rank two bundle $\mathcal{O}_{\mathrm{p}^{2}}(1) \oplus \mathcal{O}_{\mathrm{p}^{2}}(1)$ (see [3], Corollary 5.2). Now we study the case when the projected surface has just one double point.

The paper is structured as follows. In the Preliminaries we fix the notation and state the double point formula for surfaces in $G(1,3)$. In Section 2 we give the proper definitions of secant varieties in the Grassmannian context and prove Corollary 2.7, which is the starting point of the theory. In Section 3 we present some examples of surfaces in $G(1,5)$ with one apparent double point. In Section 4 we analyse the fundamental locus of a birational morphism in order to prove the rationality (Proposition 4.5) and the normal linearity (Corollary 4.7) of surfaces in $G(1,5)$ with one apparent double point. Finally, Section 5 is devoted to state and prove the classification theorem, which is the main result of the paper.

AcKnowledgements. I would like to thank my advisor Professor Enrique Arrondo for suggesting this research, following its development and for many fruitful discussions. I would also like to thank Professor Kristian Ranestad for useful remarks and comments during my stay in Oslo. Finally, it is a pleasure to thank the University of Oslo and its Department of Mathematics for its warm hospitality while this last version was written.

\section{Preliminaries}

Throughout this work $X$ will be a smooth irreducible surface over C embedded in $G(1,5)$, the Grassmann variety of lines in $\mathrm{P}^{5}$. According to this notation, $G(k, n)$ will be the Grassmann variety of $k$-planes in $\mathrm{P}^{n}$.

For $Y \subset G(k, n)$, we will denote by $\bar{Y} \subset \mathrm{P}^{n}$ the union of its $k$-planes inside $\mathrm{P}^{n} . Y$ is said to be nondegenerate if $\bar{Y}$ is not contained in any hyperplane of $\mathrm{P}^{n}$. In particular, $X \subset G(1,5)$ is always supposed nondegenerate.

A different problem would be to study surfaces in $G(1,4)$ with one apparent double point (cf. [3], Theorem 5.1 and Corollary 5.2).

We will denote the elements of $G(1, n)$ by small letters, say $l$, and use the corresponding capital letter, say $L$, for the line in $\mathrm{P}^{n}$ that they define. We usually call a line of $X \subset G(1, n)$ to refer to a line in $\mathrm{P}^{n}$ given by the corresponding point of $X$.

By (linear) projection $G(1, n) \rightarrow G\left(1, n^{\prime}\right)$ we mean the rational map induced by a linear projection $\mathrm{P}^{n} \rightarrow \mathrm{P}^{n^{\prime}}$. Its indeterminacy locus consists of the set of lines meeting the center of projection from $\mathrm{P}^{n}$ to $\mathrm{P}^{n^{\prime}}$.

For $G=G(1, n)$, we denote by $A(G)$ the Chow ring of $G$. For $0 \leq \alpha_{1}<\alpha_{2}$, $\Omega\left(\alpha_{1}, \alpha_{2}\right)$ represents the class of the Schubert variety of lines contained in $\mathrm{P}^{\alpha_{2}} \subset \mathrm{P}^{n}$ and meeting $\mathrm{P}^{\alpha_{1}} \subset \mathrm{P}^{\alpha_{2}}$. 
The rational equivalence class of $X \subset G$ in $A(G)$ is $[X]=a \Omega(0,3)+$ $b \Omega(1,2)$, where $a$ is the number of lines of $X$ meeting a general $(n-3)$ linear space of $\mathrm{P}^{n}$ and $b$ is the number of lines of $X$ contained in a general hyperplane of $\mathrm{P}^{n}$. The degree of $X$, considered as a projective surface via the Plücker embedding, is $a+b$.

For short, we will say that $X$ has bidegree $(a, b)$, where $a$ and $b$ are called respectively order and class of $X$. If $X$ has bidegree $(a, b)$, then its general projection to $G(1,3)$ has also bidegree $(a, b)$.

Let $X \subset G(1, n)$ be a surface of order $a>0$. If $\bar{X} \subset \mathrm{P}^{n}$ has degree $d$, then $a=d \cdot \mu$, where $\mu$ is the number of lines of $X$ passing through the general point of $\bar{X}$. In particular, $d \leq a$.

By $g_{X}$ we denote the sectional genus of a surface $X \subset G(1, n)$, considered as a projective variety via the Plücker embedding. If $\mu=1$, then $g_{X}$ coincides with the arithmetic genus of the curve obtained by intersecting $\bar{X} \subset \mathrm{P}^{n}$ with a general $\mathrm{P}^{n-2}$.

Following the notation of [7], for any $e \geq 0$, let $X_{e}$ be the rational ruled surface of invariant $e$.

For $a_{2} \geq a_{1} \geq 0$, we denote by $S\left(a_{1}, a_{2}\right) \subset \mathrm{P}^{a_{1}+a_{2}+1}$ the rational normal scroll of lines. This is the surface obtained by joining corresponding points of two rational normal curves $C_{a_{1}}$ and $C_{a_{2}}$. It is well known that $S\left(a_{1}, a_{2}\right)$ is an embedding of $X_{a_{2}-a_{1}}$ if $a_{1}>0$.

\subsection{Embeddings in $G(1, n)$}

Let $X$ be a smooth variety. Giving a nondegenerate morphism $f: X \rightarrow$ $G(1, n)$ is equivalent to giving a rank two bundle $\mathscr{F}$ on $X$ and an epimorphism $\phi: V \otimes \mathscr{O}_{X} \rightarrow \mathscr{F}$, where $V \subset H^{0}(X, \mathscr{F})$ is a vector subspace of dimension $n+1$ (see [3]). The second condition is simply saying that $\mathscr{F}$ is generated by global sections. In this situation $\mathscr{F} \cong Q_{\mid X}$, where $Q$ denotes the universal quotient bundle of $G(1, n)$.

Moreover, $f$ is an embedding if any subscheme of length two of $X$ imposes at least three conditions to $V$. That is, different points of $X$ (maybe infinitely close) are mapped by $f$ to different lines, since their span in $\mathbf{P}^{n}$ is at least a plane.

When $f$ is an embedding, its composition with the Plücker embedding turns out to be a projective embedding of $X$ that can be very degenerate. In fact, all the examples $X \subset G(1,5)$ of Section 3 are contained in a proper subspace of $\mathrm{P}^{14}$.

Definition 1.1. A subvariety $X \subset G(1, n)$ is said to be linearly normal if it is not isomorphically projected from any nondegenerate subvariety of $G(1, n+1)$. Since a linear projection is given by taking a linear subspace of $H^{0}(X, \mathscr{F})$, this condition is equivalent to consider $V \cong H^{0}(X, \mathscr{F})$. 
REMARK 1.2. If $\mathscr{F}$ splits as a direct sum of line bundles, say $\mathscr{F}=\mathscr{L}_{1} \oplus \mathscr{L}_{2}$, and $V=H^{0}(X, \mathscr{F})$, then $X$ has a nice geometric description. Since $\mathscr{L}_{i}$ are also generated by global sections, we have morphisms $f_{i}: X \rightarrow \mathrm{P}^{l_{i}}$. Moreover $\mathrm{P}^{l_{1}}$ and $\mathrm{P}^{l_{2}}$ span $\mathbf{P}^{n}$. Thus $f(x) \in G(1, n)$ is the line generated by $f_{1}(x)$ and $f_{2}(x)$.

\subsection{The double point formula}

A birational morphism onto its image, $X \rightarrow G(1,3)$, is expected to produce a finite number $\delta(X)$ of double points. Properly counted, it is given by the identity

$$
\delta(X)=\frac{1}{2}\left([X]^{2}-c_{2}(\mathcal{N})\right),
$$

where $[X]$ denotes the class of $X$ in $A(G)$ and $c_{2}(\mathcal{N})$ denotes the second Chern class of the normal bundle of $X$ in $G(1,3)$ (see [6], Proposition 9.3). From (1), it follows (see [3], Proposition 2.1) the double point formula for surfaces in $G(1,3)$

$$
a^{2}+b^{2}=3(a+b)+4\left(2 g_{X}-2\right)+2 K_{X}^{2}-12 \chi\left(\mathcal{O}_{X}\right)+2 \delta(X),
$$

where $g_{X}$ is the sectional genus of $X, K_{X}^{2}$ is the self-intersection number of the canonical divisor and $\chi\left(\mathscr{O}_{X}\right)$ is the Euler characteristic of the structure sheaf of $X$.

The task consists of finding embedded surfaces in $G(1,5)$ such that linear projection from $G(1,5)$ to $G(1,3)$ defines, by restriction, a morphism $X \rightarrow$ $G(1,3)$ whose image has exactly one doble point. In particular, the invariants of $X$ satisfy the double point formula (2) with $\delta(X)=1$.

\section{Secant variety and bad planes}

Let $X \subset G(1,5)$ be a smooth surface with one apparent double point (from now on OADP). Then, for a general $l \in G(1,5)$, only one of the following conditions is satisfied:

$\left.{ }^{*}\right)$ There are exactly two skew lines of $X$ (maybe infinitely close) spanning a $\mathrm{P}^{3}$ in which $L$ is contained.

$(* *)$ There is exactly one plane intersecting $X$ in a subscheme of length two and meeting $L$.

This fact motivates the definition of secant varieties in the Grassmannian context (as it is done in [1]). First, let us show the following lemma.

Lemma 2.1. Two general lines of $X$ are skew. 
Proof. Otherwise every pair of lines of $X$ intersect. Then, all the lines of $X$ either pass through a point or are contained in a plane. In the second case $X$ would be degenerate and in the first case the projection to $G(1,3)$ is nonreduced unless $X$ is the set of lines through a point and contained in a $\mathrm{P}^{3}$, which is also degenerate.

Definition 2.2. Consider the rational map $\varphi: X \times X \rightarrow G(3,5)$ which associates to each pair of skew lines $\left(l, l^{\prime}\right)$ its linear span $\Lambda=\left\langle L, L^{\prime}\right\rangle$. We define $S(X) \subset G(3,5)$, the secant variety of $X$, to be the closure of the reduced image of this map.

Remark 2.3. Let us see that $S(X)$ is an irreducible variety of dimension 2 or 4. For $\Lambda \subset S(X)$, let $Y_{\Lambda} \subset X$ be $X \cap G(1, \Lambda)$. Suppose $\operatorname{dim} Y_{\Lambda}=1$ for $\Lambda$ general. Then the general fiber of $\varphi$ has dimension 2 and $\operatorname{dim} S(X)=2$. Otherwise $\operatorname{dim} S(X)=4$.

Definition 2.4. We define the variety of bad planes of $X$ as $B(X)=\{\pi \in$ $G(2,5) \mid$ length $(G(1, \Pi) \cap X) \geq 2\}$. In other words, $B(X)$ is the set of planes in $\mathrm{P}^{5}$ containing at least two lines of $X$. Note that this variety can be reducible.

REMARK 2.5. Bad planes correspond to secant lines to $X \subset \mathrm{P}^{14}$ contained in $G(1,5)$. In what follows, we will say that two infinitely close lines intersect if there is a bad plane $\pi \in B(X)$ meeting $X$ in two infinitely close lines, that is, if there is a tangent line to $X \subset \mathrm{P}^{14}$ contained in $G(1,5)$.

Let $I$ be the incidence variety

$$
I=\{(l, \Lambda) \mid l \in G(1, \Lambda)\} \subset G(1,5) \times S(X)
$$

with corresponding projection maps $\beta_{1}: I \rightarrow G(1,5)$ and $\beta_{2}: I \rightarrow S(X)$. In the following proposition we prove that the double point actually comes from condition $(*)$.

Proposition 2.6. Let $X \subset G(1,5)$ be an OADP surface. Then $\beta_{1}: I \rightarrow$ $G(1,5)$ is dominant.

Proof. Recall that the dimension of $S(X) \subset G(3,5)$ must be 2 or 4 . If $\beta_{1}: I \rightarrow G(1,5)$ is not dominant, then it follows that $\operatorname{dim} S(X)=2$ and every $\Lambda \in S(X)$ contains a curve $Y_{\Lambda} \subset X$ (see [1], Theorem 3.1). Let us say a few words about this claim. The hypothesis of Theorem 3.1, for $n=2$, is that $X$ can be isomorphically projected to $G(1,3)$. That is, $\beta_{1}$ is not dominant and $\operatorname{dim} \overline{B(X)} \leq 3$. But, in the proof, the only condition used is that $\beta_{1}$ is not dominant, i.e., no assumptions about bad planes are needed.

If we project $X$ from a general $l \in G(1,5), L$ meets an infinite family of secant $\mathrm{P}^{3}$ 's. Hence, for a general $\Lambda, Y_{\Lambda}$ must be a (maybe reducible) curve 
that can be isomorphically projected to $G(1,2)$. Since a nondegenerate curve cannot be isomorphically projected to $\mathrm{P}^{2}$, the linear span of $Y_{\Lambda}$ under the Plücker embedding is $\mathrm{P}^{2}$. Since the Grassmannian is cut out by quadrics, $Y_{\Lambda}$ is either a smooth conic (corresponding to the lines of one ruling of a smooth quadric surface) or a singular conic (corresponding to two pencils of lines with centers in a common line).

In the first case $X$ contains a two dimensional family of smooth conics. Therefore $X$ is either a Veronese surface, a rational normal scroll of degree 3 or a quadric. But these surfaces cannot be embedded as OADP surfaces. To check this claim, note that $g_{X}=0, \chi\left(\mathscr{O}_{X}\right)=1$ and $K_{X}^{2}=9$ or 8 , contradicting the double point formula.

In the second case $X$ contains, by the Plücker embedding, a family of lines, and two of these lines always intersect. Therefore it is a cone, contradicting the smoothness of $X$.

Corollary 2.7. Let $X \subset G(1,5)$ be an OADP surface. Then the first projection $\beta_{1}: I \rightarrow G(1,5)$ is birational, length $Y_{\Lambda}=2$ for a general $\Lambda \in S(X)$ and $\operatorname{dim} \overline{B(X)} \leq 3$.

Proof. Obvious from $X$ being an OADP surface and Proposition 2.6.

\section{Examples of OADP surfaces}

Example 3.1. Consider $X_{0}=\mathrm{P}^{1} \times \mathrm{P}^{1}$ embedded in $G(1,5)$ with bidegree $(3,1)$ by the rank two bundle $\mathscr{F}=\mathscr{O}_{\mathrm{P}^{1} \times \mathrm{P}^{1}}(1,0) \oplus \mathscr{O}_{\mathrm{P}^{1} \times \mathrm{P}^{1}}(1,1)$. Since $\bigwedge^{2} \mathscr{F}=\mathscr{O}_{\mathrm{P}^{1} \times \mathrm{Pl}^{\mathrm{l}}}(2,1)$, we get the rational normal scroll $S(2,2)$ by the Plücker embedding.

In coordinates, the lines of the surface are given by the rows of the matrix

$$
\left(\begin{array}{cccccc}
x_{0} & x_{1} & 0 & 0 & 0 & 0 \\
0 & 0 & x_{0} y_{0} & x_{0} y_{1} & x_{1} y_{0} & x_{1} y_{1}
\end{array}\right) .
$$

Geometrically, we are taking a line $L$, a smooth quadric $Q$ in the complementary linear space of $\mathrm{P}^{5}$ and we fix an isomorphism between $L$ and the lines of one ruling of $Q$. Then the surface is obtained by joining each point of $L$ with points of the corresponding line of $Q$.

Example 3.2. Consider the rational ruled surface $X_{2}$ embedded in $G(1,5)$ with bidegree $(3,1)$ by the rank two bundle $\mathscr{F}=\mathscr{O}_{X_{2}}(f) \oplus \mathscr{O}_{X_{2}}\left(C_{0}+2 f\right)$. Since $\bigwedge^{2} \mathscr{F}=\mathscr{O}_{X_{2}}\left(C_{0}+3 f\right)$, we get the rational normal scroll $S(1,3)$ by the Plücker embedding.

Geometrically, we take a line $L$ (image of $X_{2}$ by $\mathscr{O}_{X_{2}}(f)$ ) and a quadric cone $C$ (image of $X_{2}$ by $\mathcal{O}_{X_{2}}\left(C_{0}+2 f\right)$ ) in the complementary linear space 
of $\mathrm{P}^{5}$. We fix an isomorphism between $L$ and the lines of the ruling of $C$. Then the surface is obtained by joining each point of $L$ with the points of the corresponding line of the ruling of $C$.

EXAmPLE 3.3. Consider the rational ruled surface $X_{1}$ embedded in $G(1,5)$ with bidegree $(3,2)$ by the rank two bundle $\mathscr{F}=\mathscr{O}_{X_{1}}(2 f) \oplus \mathscr{O}_{X_{1}}\left(C_{0}+f\right)$. Since $\bigwedge^{2} \mathscr{F}=\mathscr{O}_{X_{1}}\left(C_{0}+3 f\right)$, we obtain the rational normal scroll $S(2,3)$ by the Plücker embedding.

Geometrically, we take two skew planes $\Pi_{1}, \Pi_{2}$ in $\mathrm{P}^{5}$ and fix an isomorphism between a conic $C \subset \Pi_{1}$ (image of $X_{1}$ by $\mathscr{O}_{X_{1}}(2 f)$ ) and the pencil of lines through a point $P \in \Pi_{2}$. Note that $\Pi_{2}$ is the image of $X_{1}$ by $\mathscr{O}_{X_{1}}\left(C_{0}+f\right)$ and this map sends $C_{0}$ to $P$. We get the surface by joining each point of $C$ with points of the corresponding line of the pencil.

Example 3.4. Consider now the rational ruled surface $X_{3}$. In this case the rank two bundle giving the embedding is not decomposable. We first present a geometric construction.

Take two conics $C_{1} \subset \Pi_{1}, C_{2} \subset \Pi_{2}$ contained in two skew planes of $\mathrm{P}^{5}$. Fix an isomorphism between $C_{1}$ and $C_{2}$. Consider a point $P_{1} \in C_{1}$ and the quadric cone $Q_{P_{1}}$ with base $C_{2}$ and vertex $P_{1}$. By joining points of $C_{1}$ and points of the corresponding line of the ruling of the cone $Q_{P_{1}}$, we obtain the rational normal scroll $S(1,4)$. Note that the pencil of lines through $P_{1}$ is a line via the Plücker embedding and, similarly, the set of lines joining corresponding points of $C_{1}$ and $C_{2}$ turns out to be a rational normal cuartic. Since a general hyperplane $H \subset \mathrm{P}^{5}$ cuts $C_{1}$ in two points, and just one line of the pencil through these points is contained in $H$, we have $b=2$. Therefore $X$ is embedded with bidegree $(3,2)$.

The rank two bundle $\mathscr{F}$ giving the embedding of $X_{3}$ in $G(1,5)$ is a nontrivial extension of line bundles

$$
0 \rightarrow \mathscr{O}_{X_{3}}\left(C_{0}+2 f\right) \rightarrow \mathscr{F} \rightarrow \mathscr{O}_{X_{3}}(2 f) \rightarrow 0 .
$$

In fact, the fundamental conic $C_{1}$ produces a quotient $\mathscr{F} \rightarrow \mathscr{O}_{X_{3}}(2 f) \rightarrow 0$ whose kernel is precisely $\mathscr{O}_{X_{3}}\left(C_{0}+2 f\right)$.

Example 3.5. Let $X$ be the blow up of $\mathrm{P}^{2}$ in three points and the embedding in $G(1,5)$ with bidegree $(4,2)$ given by the rank two bundle $\mathscr{F}=\mathscr{O}_{X}(2 L-$ $\left.E_{1}-E_{2}-E_{3}\right) \oplus \mathscr{O}_{X}(L)$. Since $\bigwedge^{2} \mathscr{F}=\mathscr{O}_{X}\left(3 L-E_{1}-E_{2}-E_{3}\right), X$ is a Del Pezzo surface of degree 6 by the Plücker embedding.

Geometrically, we fix a Cremona transformation of $\mathrm{P}^{2}$. The lines of $X$ are given by joining corresponding points of two skew $\mathrm{P}^{2}$, $s$.

In coordinates, consider the Cremona transformation $\varphi: \mathbf{P}^{2} \rightarrow \mathrm{P}^{2}$ defined by $\varphi\left(x_{0}: x_{1}: x_{2}\right)=\left(x_{1} x_{2}: x_{0} x_{2}: x_{0} x_{1}\right)$. Then, the lines of the surface are 
given by the rows of the matrix

$$
\left(\begin{array}{cccccc}
x_{0} & x_{1} & x_{2} & 0 & 0 & 0 \\
0 & 0 & 0 & x_{1} x_{2} & x_{0} x_{2} & x_{0} x_{1}
\end{array}\right) .
$$

By the Plücker embedding we get a set of generators of the complete linear system of plane cubics passing through the points $\{(1: 0: 0),(0: 1: 0),(0$ : $0: 1)\}$, giving the embedding of the Del Pezzo surface of degree 6 in $P^{6}$.

EXAMPLE 3.6. We want to embed the blow up of $\mathrm{P}^{2}$ in two points in $G(1,5)$ as a Del Pezzo surface of degree 7 and bidegree $(4,3)$. First of all, consider the embedding of $Y=\mathrm{P}^{2}$ in $G(1,7)$ with bidegree $(6,3)$ given by the tangent bundle $\mathscr{T}_{\mathrm{P}^{2}}$. Geometrically, this $\mathrm{P}^{2}$ is one of the two families of lines of the threefold $\bar{Y}=\mathrm{P}\left(\mathscr{T}_{\mathrm{P}^{2}}\right)$ embedded in $\mathrm{P}^{7}$ by its tautological line bundle.

We obtain $X$, the Del Pezzo surface of degree 7 and bidegree $(4,3)$, by projecting $Y$ from a general secant line $r$ to $\bar{Y}$. The induced projection $\pi_{r}$ : $Y \rightarrow X$ is not defined in the two lines of $Y$ passing through the corresponding points of $\bar{Y}$.

To prove that $X$ is smooth, it is enough to check that $r$ is contained in just one $\mathrm{P}^{3}$ spanned by two lines of $Y$. Since $\mathscr{T}_{\mathrm{P}^{2}}$ is a uniform rank two bundle of splitting type $(2,1), \mathscr{T}_{\mathrm{P}^{2}}$ restricted to any line $L \subset \mathrm{P}^{2}$ embedds $L$ as the lines of the ruling of a rational normal scroll $S_{L}(1,2) \subset \mathrm{P}_{L}^{4}$. Consider another line $L^{\prime} \subset \mathrm{P}^{2}$ and the corresponding scroll $S_{L^{\prime}}(1,2) \subset \mathrm{P}_{L^{\prime}}^{4}$. Since $\mathrm{P}_{L}^{4} \cap \mathrm{P}_{L^{\prime}}^{4}$ is exactly the fiber of the scrolls corresponding to the point $P=L \cap L^{\prime}$, we can suppose $r$ just contained in $\mathrm{P}_{L}^{4}$. Therefore the only secant $\mathrm{P}^{3}$ to $Y$ containing $r$ is spanned by $r$ and the exceptional line of the scroll $S_{L}(1,2) \subset \mathrm{P}_{L}^{4}$.

REMARK 3.7. A straightforward computation in (2) shows that these examples actually give OADP surfaces. Moreover it is not hard to find geometrically the secant $\mathrm{P}^{3}$ containing a general $l \in G(1,5)$.

\section{Some properties of OADP surfaces in $G(1,5)$}

In this section we state some properties of OADP surfaces in $G(1,5)$ analogous to those of OADP surfaces in $\mathrm{P}^{5}$ (cf. [8] or [5]). Let us recall the following result (see, for instance, [7]).

TheORem 4.1 (Zariski's Main Theorem). Let $f: X \rightarrow Y$ be a birational projective morphism of noetherian integral schemes, and assume that $Y$ is normal. Then for every $y \in Y, f^{-1}(y)$ is connected.

Definition 4.2. Let $f: X \rightarrow Y$ be a generically finite morphism of noetherian integral schemes. We define the branch locus of $f$, denoted by $B \subset Y$, to be the image by $f$ of the ramification divisor $R$ on $X$ and the 
fundamental locus of $f$, denoted by $F \subset Y$, to be the variety of points with fibre of positive dimension.

Lemma 4.3. Let $f: X \rightarrow Y$ be a generically finite morphism of noetherian integral schemes. Then $F \subset B$ and $\operatorname{codim}_{Y} F>1$. Moreover if $f$ is birational and $Y$ normal, then $F=B$.

PRoOF. For any $y \in F$ there exists at least a curve $C \subset f^{-1}(y)$. Take a point $c \in C$. Since $d_{c} f(v)=0$ for any $v \in T_{c} C$, the differential map $d_{c} f: T_{c} X \rightarrow T_{y} Y$ is not injective and, therefore, $f(c)=y \in B$. Obviously $\operatorname{codim}_{Y} F>1$ since, otherwise, $f^{-1}(F)=X$.

Let us now suppose $f: X \rightarrow Y$ to be birational and $Y$ normal. For any $y \in B$ the morphism $f$ is not an isomorphism in $f^{-1}(y)$ and hence length $\left(f^{-1}(y)\right) \geq 2$. By Zariski's Main Theorem $4.1 y$ is a fundamental point, i.e., $y \in F$.

Consider again the incidence variety

$$
I=\{(l, \Lambda) \mid l \in G(1, \Lambda)\} \subset G(1,5) \times S(X)
$$

with corresponding projection maps $\beta_{1}: I \rightarrow G(1,5)$ and $\beta_{2}: I \rightarrow S(X)$. Let $R_{\Lambda}=\{(r, \Lambda) \mid(r, \Lambda) \in R\}$ be the restriction to $G(1, \Lambda) \times \Lambda$ of the ramification divisor $R$. Then $R_{\Lambda}$ is a divisor on $G(1, \Lambda) \times \Lambda$ for a general $\Lambda \in S(X)$. By Corollary 2.7 we know that $\beta_{1}$ is birational and, by Lemma 4.3, $\beta_{1}(R)=F$.

Denote by $\Gamma_{X} \subset G(1,5)$ the variety of lines meeting $\bar{X}$ and $\Sigma_{X} \subset \Gamma_{X}$ the (usual) secant variety of $\bar{X} \subset \mathrm{P}^{5}$. Both are, respectively, irreducible varieties of dimension 7 and 6 .

Lemma 4.4. Neither $\Gamma_{X}$ nor $\Sigma_{X}$ are contained in the fundamental (or branch) locus $F$ of $\beta_{1}: I \rightarrow G(1,5)$.

Proof. By counting dimensions, $\Gamma_{X}$ is not contained in $F$. On the other hand, if $\Sigma_{X} \subset F$, then $\Sigma_{X}$ is a component of maximal dimension of $F$. Thus, for a general $\Lambda \in S(X), R_{\Lambda}$ contains a 3-dimensional subscheme $\Sigma_{X, \Lambda} \subset \Sigma_{X}$. Since $\Sigma_{X, \Lambda}$ is precisely the (usual projective) secant variety of $\bar{X} \cap \Lambda$, it has even dimension. Therefore $\Sigma_{X}$ cannot be contained in $F$.

We can now prove the first interesting property of OADP surfaces in $G(1,5)$.

Proposition 4.5. OADP surfaces in $G(1,5)$ are rational.

Proof. Let $l \in X$ be a general line. Consider the inner projection $\pi_{l}$ : $X \rightarrow G(1,3)$ and denote by $X^{\prime}$ the image of $X$ under this projection. We claim that $X^{\prime}$ is a congruence of lines of order one. If through the general 
$P \in \mathrm{P}^{3}$ there pass more than one line of $X^{\prime}$, the plane $\langle P, L\rangle \subset \mathrm{P}^{5}$ is cut by at least two lines of $X$ outside $L$. Hence, a line $r \in G(1,\langle P, L\rangle)$ is contained in a subscheme of $S(X)$ of length at least two. By Theorem 4.1, $r$ is contained in the fundamental locus $F$ of $\beta_{1}: I \rightarrow G(1,5)$. But lines $r \in G(1,\langle p, L\rangle)$ sweep out $\Gamma_{X}$ when we move $l$ and $P$, contradicting Lemma 4.4.

A congruence of order one $X^{\prime} \subset G(1,3)$ is necessarily rational. The birational morphism to $P^{2}$ is given by intersecting the lines of $X^{\prime}$ with a fixed plane of $\mathrm{P}^{3}$. Since length $Y_{\Lambda}=2$ by Corollary 2.7, $\pi_{l}: X \rightarrow X^{\prime}$ is a birational map and $X$ is also a rational surface.

Remark 4.6. The proof of Proposition 4.5 resembles the proof in the projective case. A surface $X \subset \mathrm{P}^{5}$ can be projected to $\mathrm{P}^{2}$ from a general tangent plane to $X$ and this projection turns out to be birational (see [5], Corollary 4.2). On the other hand we are projecting $X$, via the Plücker embedding, from $\left\langle\Omega\left(L, \mathrm{P}^{5}\right)\right\rangle \cong \mathrm{P}^{8}$. But $\left\langle\Omega\left(L, \mathrm{P}^{5}\right)\right\rangle$ is precisely the tangent space to $G(1,5)$ at the point $l \in X$.

Corollary 4.7. OADP surfaces in $G(1,5)$ are linearly normal.

Proof. Let $X \subset G(1,6)$ be a nondegenerate OADP surface that can be isomorphically projected to $G(1,5)$ from a general point $P \in \mathrm{P}^{6}$. From the proof of Proposition 4.5, it follows that the further inner projection $\pi_{l}: X-\rightarrow$ $G(1,3)$ from a general line $l \in X$ gives rise to a congruence of order one in $G(1,3)$. But projecting from $P$ and then projecting from $L$ is equivalent to projecting from $L$ and then projecting from $P$. Therefore the inner projection $\pi_{l}(X) \subset G(1,4)$ is a surface of order one. Since $a=1$, then $d=1$ and the threefold covered by the lines of $\pi_{l}(X)$ is a hyperplane in $\mathrm{P}^{4}$. Hence $X \subset$ $G(1,6)$ is degenerate, which is a contradiction.

\section{The classification theorem}

In this section we state and prove the main result on OADP surfaces in $G(1,5)$, namely the classification theorem.

Theorem 5.1. Let $X \subset G(1,5)$ be a smooth OADP surface. Then $X$ is either a rational scroll embedded with bidegree $(3,1)$ or $(3,2)$ or a Del Pezzo surface embedded with bidegree $(4,2)$ or $(4,3)$.

We divide the proof of the theorem in two different cases, according to the fact that the general line of $X$ can be intersected by another line of $X$ or not. Hence the proof of the theorem will be a consequence of the following two propositions.

Proposition 5.2. Let $X \subset G(1,5)$ be an OADP surface. Suppose that the general line of $X$ intersects another line of $X$ (maybe infinitely close). Then $X$ is a rational scroll embedded with bidegree $(3,1)$ or $(3,2)$. 
Proof. If a general line of $X$ intersects another line of $X$, then $\bar{X} \subset \overline{B(X)}$. Let $B$ be an irreducible component of $B(X)$. If $\operatorname{dim} B \geq 2$, since $\operatorname{dim} \bar{B} \leq 3$ by Lemma 2.7, it follows that $\bar{B}=\mathrm{P}^{3}$ and then $\bar{X} \not \subset \bar{B}$. So a general $l \in X$ is not contained in a plane of $B$. Since $\bar{X} \subset \overline{B(X)}$, there exists a component $B^{\prime} \subset B(X)$ of dimension one such that $\bar{X}=\bar{B}^{\prime}$. Hence, $\bar{X}$ is a scroll of planes over a rational curve (by Proposition 4.5) and every plane $\Pi$ of the family contains infinitely many lines of $X$.

The set of lines of $X$ in $\Pi$ is a pencil, i.e., $\mu=1$. Otherwise a general $r \in \Sigma_{X}$ is contained in more than one secant $\mathrm{P}^{3}$ and $\Sigma_{X}$ should be contained in the fundamental locus of $\beta_{1}: I \rightarrow G(1,5)$, contradicting Lemma 4.4. Therefore $X$ is a rational scroll, so $g_{X}=0, K_{X}^{2}=8$ and $\chi(X)=1$. The double point formula (2) becomes $(2 a-3)^{2}+(2 b-3)^{2}=10$, whose solutions are $\{(0,1),(1,0),(0,2),(2,0),(1,3),(3,1),(2,3),(3,2)\}$. But $\bar{X} \subset \mathrm{P}^{5}$ is a nondegenerate 3 -fold, so $d=\operatorname{deg} \bar{X} \geq 3$ and then $a=3$.

Proposition 5.3. Let $X \subset G(1,5)$ be an OADP surface. Suppose that the general line of $X$ does not intersect another line of $X$ (neither infinitely close). Then $X$ is a Del Pezzo surface embedded with bidegree $(4,2)$ or $(4,3)$.

Proof. In order to prove this proposition we will need the following two lemmas.

Lemma 5.4. The singular points of the Schubert variety $\Omega\left(\mathrm{P}^{2}, \mathrm{P}^{5}\right)$, corresponding to the lines inside the plane, have multiplicity 3.

Proof. The open affine subset $U_{0,1} \subset G(1,5)$ is given by the lines generated by the rows of the matrix

$$
\left(\begin{array}{llllll}
1 & 0 & a_{2} & a_{3} & a_{4} & a_{5} \\
0 & 1 & b_{2} & b_{3} & b_{4} & b_{5}
\end{array}\right)
$$

A line meeting the plane $\Pi$ of equations $\left\{x_{3}=x_{4}=x_{5}=0\right\}$ is characterized by the condition

$$
\operatorname{rank}\left(\begin{array}{ccc}
a_{3} & a_{4} & a_{5} \\
b_{3} & b_{4} & b_{5}
\end{array}\right) \leq 1
$$

and we get a line of the plane $\Pi$ when the rank of the above matrix is zero. Then, we have to compute the multiplicity of the zero matrix in the determinantal variety of matrices $2 \times 3$ of rank less than or equal to one. This multiplicity is the degree of the tangent cone, i.e., the degree of the variety defined by the ideal generated by $\left\{a_{3} b_{4}-a_{4} b_{3}, a_{3} b_{5}-a_{5} b_{3}, a_{4} b_{5}-a_{5} b_{4}\right\}$ in $k\left[a_{3}, a_{4}, a_{5}, b_{3}, b_{4}, b_{5}\right]$. This is precisely the ideal of the Segre embedding of $P^{1} \times P^{2}$ in $P^{5}$, and its degree is 3 . 
Lemma 5.5. Let $X \subset G(1,5)$ be a surface of bidegree $(a, b)$ such that the general $l \in X$ does not intersect any other line of $X$ (neither infinitely close). Then the inner projection $X^{\prime}=\pi_{l}(X)$ has bidegree $\left(a^{\prime}, b^{\prime}\right)=(a-3, b-1)$ in $G(1,3)$.

Proof. Let us see that $b^{\prime}=b-1$, where $b^{\prime}$ is the set of lines of $X^{\prime} \subset G(1,3)$ contained in a general plane. Note that we can choose a plane $\Pi \subset \mathrm{P}^{3}$ such that $K=\langle L, \Pi\rangle$ is a $\mathrm{P}^{4}$ which does not contain any infinitely close secant $\mathrm{P}^{3}$ spanned by $l$. Then $G(1, K)$ and $X$ meet transversally at $l$, so $l$ should be counted with multiplicity one.

Now take $P \in \mathrm{P}^{3}$. In this case, $a^{\prime}$ is the number of lines of $X^{\prime}$ through $P$, which corresponds to lines of $X$ meeting $\Pi=\langle P, L\rangle$. By Lemma 5.4, $\Omega\left(\Pi, \mathrm{P}^{5}\right)$ is singular along $G(1, \Pi)$ with multiplicity 3 . To prove that the intersection multiplicity of $X$ and $\Omega\left(\Pi, \mathrm{P}^{5}\right)$ along $l$ is exactly 3 is equivalent to check that no line of the tangent cone to $\Omega\left(\Pi, \mathrm{P}^{5}\right)$ at $l$ is also tangent to $X$ (see [6], Corollary 12.4). But lines of the tangent cone to $\Omega\left(\Pi, \mathrm{P}^{5}\right.$ ) at $l$ correspond to pencils of lines through a point of $L$ in a plane containing $L$. By hypothesis, no such pencil can meet $X$ at two lines, so $l$ must be counted with multiplicity 3 .

Let us now prove Proposition 5.3. From Lemma 5.5 and the claim of Proposition 4.5 , it follows that, under this hypothesis, the order of $X$ is 4 . Let us see that $g_{X}=1$. Since a general line of $X$ does not intersect any other line, $\bar{X} \subset \mathrm{P}^{5}$ cannot be a cone. By the same reason, $\mu=1$. Hence $\bar{X}$ has degree 4 and it must be the complete intersection of two quadrics in $\mathrm{P}^{5}$. Recall that $g_{X}$ is the arithmetic genus of the curve obtained by intersecting $\bar{X}$ with a general $\mathrm{P}^{3}$, so $g_{X}=1$ and $X$ is necessarily a Del Pezzo surface. Therefore $K_{X}^{2}=a+b$ and $\chi(X)=1$, so the double point formula (2) becomes $(2 a-5)^{2}+(2 b-5)^{2}=10$. Since $a=4$, its solutions are $(4,2)$ or $(4,3)$.

\section{REFERENCES}

1. Arrondo, E., Projections of Grassmannians of lines and characterization of Veronese varieties, J. Algebraic Geom. 8 (1999), 85-101.

2. Arrondo, E., Sierra, J. C., and Ugaglia, L., Classification of $n$-dimensional subvarieties of $G(1,2 n)$ that can be projected to $G(1, n+1)$, preprint 2003.

3. Arrondo, E., and Sols, I., On congruences of lines in the projective space, Mém. Soc. Math. France (N.S) 50 (1992).

4. Aure, A., The smooth surfaces in $\mathrm{P}^{4}$ without apparent triple points, Duke Math. J. 57 (1988), 423-430.

5. Ciliberto, C., Mella, M., and Russo, F., Varieties with one apparent double point, J. Algebraic Geom. 13 (2004), 475-512.

6. Fulton, W., Intersection Theory, Ergeb. Math. Grenzgeb. (3) 2 (1984).

7. Hartshorne, R., Algebraic Geometry, Grad. Texts in Math. 52 (1977). 
8. Russo, F., On a theorem of Severi, Math. Ann. 316 (2000), 1-17.

9. Severi, F., Intorno ai punti doppi impropri di una superficie generale dello spazio a quattro dimensioni e ai suoi punti tripli apparenti, Rend. Circ. Mat. Palermo 15 (1901), 33-51.

10. Ugaglia, L., Subvarieties of the Grassmannian $G(1, N)$ with small secant variety, Comm. Algebra 30 (2002), 4059-4083.

DEPARTAMENTO DE ÁLGEBRA

FACULTAD DE CIENCIAS MATEMÁTICAS

UNIVERSIDAD COMPLUTENSE

28040 MADRID

SPAIN

E-mail: jcsierra@mat.ucm.es 